\title{
WiFi-enabled Automatic Eating Moment Monitoring Using Smartphones
}

\author{
Zhenzhe Lin ${ }^{1}$, Yucheng Xie ${ }^{2}$, Xiaonan Guo ${ }^{2}$, Chen Wang ${ }^{3}$, Yanzhi Ren ${ }^{4}$, and \\ Yingying Chen ${ }^{1}$ \\ 1 WINLAB, Rutgers University, New Brunswick, NJ, USA 08901 \\ zhenzhe.lin@rutgers.edu, yingche@scarletmail.rutgers.edu \\ 2 Indiana University-Purdue University Indianapolis, IN, USA 46202 \\ yx11@iupui.edu, xg6@iupui.edu \\ 3 Louisiana State University, Baton Rouge, LA, USA 70803 \\ chenwang1@lsu.edu \\ 4 University of Electronic Science and Technology of China, P.R. China 611731 \\ renyanzhi05@uestc.edu.cn
}

\begin{abstract}
Dietary habits are closely correlated with people's health. Study reveals that unhealthy eating habits may cause various diseases such as obesity, diabetes and anemia. To help users create good eating habits, eating moment monitoring plays a significant role. However, traditional methods mainly rely on manual self-report or wearable devices, which either require much user efforts or intrusive dedicated hardware. In this work, we propose a user effort-free eating moment monitoring system by leveraging the $\mathrm{WiFi}$ signals extracted from the commercial off-the-shelf (COTS) smartphones. In particular, our system captures the eating activities of users to determine the eating moments. The proposed system can further identify the fine-grained food intake gestures (e.g., eating with fork, knife, spoon, chopsticks and bard hand) to estimate the detailed eating episode for each food intake gesture. Utilizing the dietary information, our system shows the potential to infer the food category and food amount. Extensive experiments with 10 subjects over 400-minute eating show that our system can recognize a user's food intake gestures with up to $97.8 \%$ accuracy and estimate the dietary moment within 1.1-second error.
\end{abstract}

Keywords: Eating moment monitoring $\cdot$ WiFi sensing $\cdot$ Healthy eating

\section{Introduction}

Dietary behavior is an important factor of healthy eating and is closely related to the health condition of an individual. Due to the increasing stress at work and the fast-paced lifestyle in the modern society, people tend to form unhealthy eating habits unconsciously, such as overeating and eating disorder, leading to weight-gain or obesity. Studies show that overweight and obesity are the most prevalent health problems, which further cause various diseases such as diabetes, high blood pressure, cardiovascular diseases and breathing disorder [1,2]. Recent

This is the author's manuscript of the article published in final edited form as:

Lin, Z., Xie, Y., Guo, X., Wang, C., Ren, Y., \& Chen, Y. (2020). Wi-Fi-Enabled Automatic Eating Moment Monitoring Using Smartphones. In N. M. Garcia, I. M. Pires, \& R. Goleva (Eds.), IoT Technologies for HealthCare (pp. 77-91). Springer International Publishing. https://doi.org/10.1007/978-3-030-42029-1_6 
survey by the World Health Organizations reveals that more than 1.9 billion adults are overweight and 650 million are obese [3], and they are suffering or under the risk of the various eating-related health problems. Therefore, it is crucial to provide an appropriate assistance to each individual for improving their dietary behaviors. To achieve this goal, we need to help the user to keep close track of every moment of his/her eating activities.

Eating moment monitoring provides the comprehensive dietary information to help users understand their eating behaviors (e.g., overeating, undereating, skipping meals, irregular eating schedules and eating too fast). Based on that, users could realize the overall health condition in regard to his/her eating behavior and further boost their ability to create and sustain a healthy lifestyle. Traditional eating moment or eating episode monitoring mainly rely on the self-reports including food questionnaires and meal records [4]. For example, a mobile application called ate [5] allows users to track their dietary information regarding the food category by capturing the food photos from cameras. However, self-report approaches require proactive participation and self-consciousness which seems to be obtrusive for the users. Moreover, these instruments suffer from subjective bias and memory imprecision [6]. To provide automatic eating moment monitoring without requiring much user efforts, recent studies propose to leverage the wearable devices worn on the user's ear [7] or wrist [8] for dietary activity recognition. Unfortunately, there are some limitations since the dedicated wearable devices also incur high-cost issues and bring additional uncomfortable user experience to individuals. Different from the above studies, in this work, we propose to leverage the WiFi signals extracted from the user's smartphones to provide fine-grained dietary moment monitoring. While eating, user can simply place his/her personal smartphone on the dining table, which automatically recognizes the food intake gestures to monitor the user's eating moments.

Recent years have witnessed the initial success of WiFi-based human activity sensing [9-11]. But in order to utilize the WiFi signals to automatically recognize the user's dietary moments, a number of challenges need to be addressed: 1) to monitor the user's eating moments, we need to recognize the user's eating activities from the many other daily activities. But it is hard for a smartphone to store a large activity profile covering all the user's daily activities such as typing, reading, sitting and stretching; 2) besides recognizing the coarse eating moments, fine-grained eating moment monitoring also require differentiating the user's various food intake gestures (e.g., eating with a folk, chopsticks or bare hand), which reflects the user's detailed eating behavior and information of the food. But the various eating gestures are similar and hard to be distinguished from the WiFi signals, which all involve the hand movements from the table to mouth; 3) using the WiFi signals from the smartphone to provide human activity recognition is still an open area. This is because the WiFi signals obtained by the smartphone is relatively weak and noisy due to the integrated small size of internal antennas.

To address these challenges, we propose a WiFi-enabled automatic dietary moment recognition system for assisting the individuals to improve dietary be- 
haviors by using their own smartphones. We extract the Channel State Information (CSI) from the WiFi signals to capture the user's fine-grained eating activities and estimate the detailed eating moments, which is non-invasive to the user and does not require additional hardware. Specifically, we utilize a Fuzzy C-Means clustering method to distinguish the dietary activities from the many other human daily activities and detect the dietary moments based on deriving the CSI spectrogram. Moreover, we extract the unique features to capture the eating gestures' behavioral characteristics and further classify them based on the utensils held by the user (i.e., fork, knife, spoon, chopsticks and bare hand). In addition, we derive the starting and ending point of the eating moment for each eating gesture and estimate the duration and speed for meals.

\section{Our contributions are summarized as follows:}

- We demonstrate that the channel state information extracted from the WiFi signals can be used to provide fine-grained eating moment monitoring for the users, which can further interpret the user's eating behavior, including overeating, undereating, eating disorder and eating too fast.

- We develop a device-free dietary recognition system based on the WiFi signals to automatically track the user's eating activity, which can be easily deployed on the user's smartphone without incurring additional costs or changing the existing WiFi infrastructures.

- We adopt the Fuzzy C-means clustering technique to differentiate the dietary activities from all human daily activities. We then utilize different machine learning classification approaches (i.e., Random Forest, Naive Bayes, K Nearest Neighbors, Discriminant Analysis Classifier) to identify the food intake gestures based on the utensils held by users. Moreover, we propose an intake gesture density derivation method to calculate the comprehensive dietary moments and develop the ingestion period estimation method to derive the dietary moment statistics.

- Extensive experiments with 10 people over 400-minute eating show that our system can recognize a user's food intake gestures with up to $97.8 \%$ accuracy and estimate the dietary moments within 1.1 second error.

\section{Related Work}

The exploit of dietary monitoring methods provokes the feasibility to infer the health condition of subjects based on eating. Traditional eating monitoring methods are mainly based on self-report or meal recalls. These methods require users to manual write down the start/end time of their eating activities on food diaries or fill questionnaires by recalling their memory [12]. The smartphone Apps allows the user to more flexibly record their meal by typing texts and taking photos [5]. But these self-report methods rely too much on the user's active participation and impose a memory burden. Thus these methods are obtrusive to the users and hard to obtain the timely precise eating monitoring result. Moreover, these methods suffer from the subjective bias and memory recall imprecision. 
To reduce the user's efforts, the vision-based methods are developed to recognize eating activities automatically by utilizing the cameras to take photos or videos of the user's meals [13]. For example, O'Loughlin et al. [14] examine the feasibility of utilizing the Microsoft SenseCam [15] (i.e., a wearable camera) to estimate the dietary energy intake within various sporting populations. However, these approaches may raise some privacy concerns due to the fact that camera could capture the user's personal sensitive information such as social relationships (i.e., eating with whom) and location privacy (i.e., where).

Recently, some wearable device based approaches have been proposed to detect the user's eating periods by leveraging the embedded sensors (e.g., microphones and motion sensors). Sazonov et al. [7] develop a system utilizing the piezoelectric sensor attached to the ear to detect the chewing and swallowing. Similarly, Bedri et al. [16] design an eating episode detection system by utilizing a dedicated ear-worn device, which is equipped with an inertial sensor behind the ear to detect people's dietary motions. Rather than the ear-worn sensor, Thomaz et al. [8] use the accelerometer on a smartwatch to infer eating moments to capture the user's hand motions during dietary period. Along this direction, Zhang et al. [17] propose a dedicated wearable device using wireless accelerometers attached on both wrists of users to detect the eating/drinking activities based on the three-dimensional kinematics movement model. Unfortunately, these wearable-based methods requiring the user to equip with dedicated hardware platforms during eating, which are obtrusive to users and also shows the limitation of their deployments in the practical scenarios.

Our work is different in that we propose a low user-effort dietary moments recognition system by leveraging the WiFi signals extracted from the user smartphone, which is a pervasive mobile device. Our system is low-cost and easy-to-use without additional dedicated devices or professional installations. Specifically, the proposed system could detect the eating episode on a daily life, including the starting/ending time of each meal, which provides an automatic solution to track the user's dietary schedule. Moreover, the system could identify the utensils held by the participants (e.g., folk and spoon) during the intake period and further segment the dietary moments into detailed eating episodes according to different utensils. Based on the comprehensive dietary information, it shows the potential to further infer how and what the users eat and measure the dietary behaviors of users.

\section{System and Methodology}

\subsection{System Overview}

The main goal of our work is to let user simply place his/her smartphone on the dinning table to achieve automatic fine-grained eating moment monitoring. The basic idea is to recognize the dietary moments by extracting the unique physiological and behavioral characteristics inherited from food intake gestures by leveraging the readily available WiFi signals. As illustrated in Figure 1, the system takes the CSI measurements extracted from the personal mobile devices 


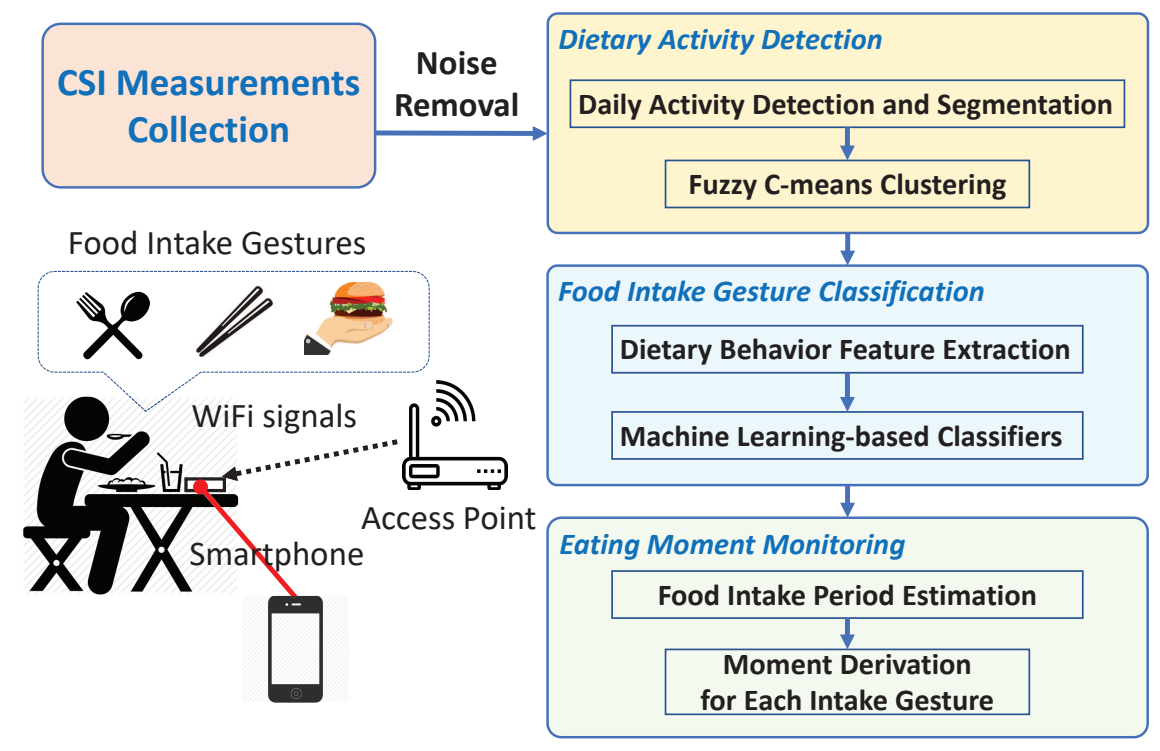

Fig. 1. Overview of the proposed system for eating moment monitoring.

(i.e., smartphones) as the input. To mitigate the ambient noises and reduce the influence of wireless interference, we apply Noise Removal techniques to remove the outliers of CSI raw data and reduce the high and low frequency noises using a bandpass filter. Next, our system performs Dietary Activity Detection to distinguish the relative eating activities from many other daily human activities (e.g, walking, reading, talking) in the environment. Specifically, we examine the moving variance and accumulated short time energy (STE) of the calibrated CSI data to obtain the segments containing the user's daily activities. We then develop the Fuzzy C-means Clustering method to recognize the dietary activities in a cluster and differentiating them from non-eating activities based on calculating the Euclidean distance between eating cluster center and the test activity. To further identify the specific food intake gestures (i.e., eating with fork, spoon, knife, chopsticks and bare hands) of the user, the system extracts unique features from both time and frequency domains to capture the inherent physiological and behavioral characteristics of user's motions. Base on the extracted features, we perform Food Intake Gesture Classification to recognize the user's different eating gestures. We test several different machine learning classifiers including Random Forest (RF), Naive Bayes (NB), K Nearest Neighbors (KNN), Discriminant Analysis Classifier (DAC), respectively. The last component Eating Moment Estimation estimates the food intake period of the user to infer the eating schedule and further divide the eating moments according to different eating gestures to estimate eating episode of each gesture. The eating gestures reflect how and what the user eats, for example eating steak with knife 
and a burger with bare hand. In addition, the number of each eating gesture is derived, which helps to know how much the user eats as well as how fast the user eats.

\subsection{Data Collection and Noise Removal}

CSI measurements are readily available from some commodity WiFi network interface controller (NIC) such as the Intel 5300 NIC. In order to perform automatic eating moment monitoring, we utilize the CSI measurements extracted from a subject's smartphone, to capture the minute differences of the channel state variations induced by a subject's food intake gestures. The intuition is that CSI measurement describes how a WiFi signal propagates over multiple subcarriers from a pair of transmitter and receiver. In addition, it represents the combined effect of scattering, fading, and power decay with distances. Specially, the CSI measurements in regard to each subcarrier can be denoted as:

$$
H_{k}=\left|H_{k}\right| e^{j \angle H_{k}}
$$

where $\left|H_{k}\right|$ and $\angle H_{k}$ describe the corresponding amplitude and phase. They represent the signal interference impacted by the human body movements, including absorption, reflection and refraction by food intake gestures. However, a subject's eating activity is very complicated since people perform different ways using their arm to take food from plate to mouth. Besides, the intake process may involve different utensils (i.e., spoon, fork, knife, and chopsticks), increasing the difficulty to perform fine-grained eating recognition. In that case, we need to extract more information based on the raw CSI measurements to provide an accurate description of the wireless signals.

Since the existing of ambient noises in daily dining environments, the WiFi signals also suffer from signal scattering and wireless interference. To mitigate the ambient noises on the CSI measurements, our system first applies a bandpass filter to remove the noises of high-frequency and low-frequency to ensure the reliability of WIFI signals. According to our observations the frequency of relative environment noises usually present in a fixed frequency range, we thus utilize an empirical threshold to remove the ambient noises.

\subsection{Dietary Activity Detection}

Daily Activity Detection and Segmentation After removing the irrelevant ambient noises, we perform daily activity detection and segmentation on the CSI measurements that mapping the wireless signal with the people's daily activities. Inspired by experiment observations, we find that human activities (i.e., walking, standing) involved the body movements lead to some vibrations on the CSI raw data. However, the variances caused by body movements usually present as a non-obvious pattern and hard to be detected. Moreover, dietary activities (e.g., using fork to bring food from dining table to mouth) usually involved minute scale body movements than walking activities, which increases the difficulty of detection. To enlarge the variances in original CSI measurements, we thus adopt 


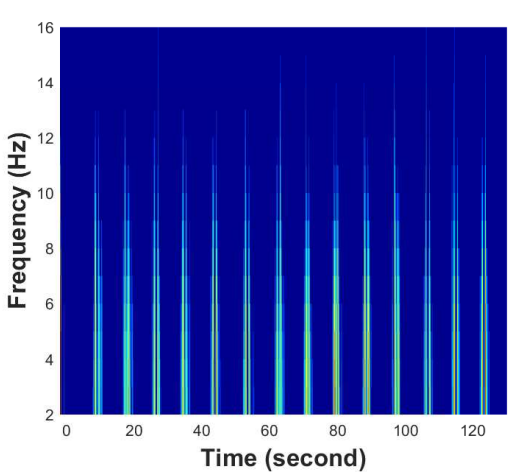

Fig. 2. Accumulated power spectral density of CSI in frequency domain.

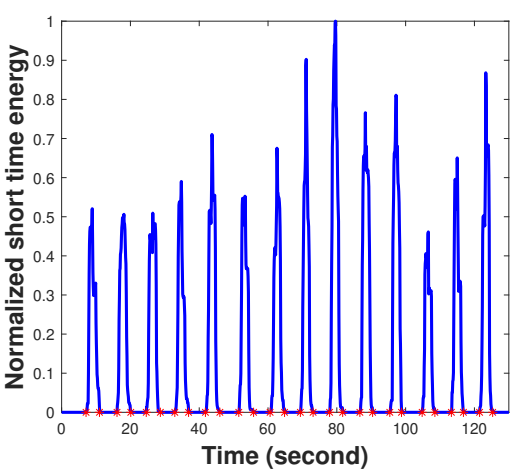

Fig. 3. Illustration of the STE-based activity detection and segmentation.

a spectrogram-based approach to calculate the short time energy (STE) upon CSI amplitude's moving variance to detect human activity. As shown in Figure 2, we derive the STE by calculating the integration of power spectral density along the frequency domain. Additionally, STE approach is more sensitive to human body movements by reconstructing the minute motions within a sliding window, which also enlarges the variances in frequency domain upon CSI measurements. We calculate STE by the following formula:

$$
S T E(\delta)=\sum_{i=1}^{I}[P S D(\delta) W(\delta+i)]^{2},
$$

where $P S D(\delta)$ represents the power spectral density function, $W(\delta)$ denotes the window function and $I$ is the length of the sliding window. Figure 3 shows the segments of human activities (i.e., use bare hands to bring food to mouth). It is obvious that STE demonstrated as great values when the dietary activity occurs. Moreover, we found there are some peaks always locate at the center of the activity duration. Inspired by this, we define the two adjacent zero points to segment the corresponding human activities.

Fuzzy C-means Clustering After detecting all of the daily activities from users, we apply an unsupervised clustering method to further differentiate the related dietary activities from the human daily activities. The basic idea is the dietary activities are defined as the arm and hand gestures involved in bringing food to the mouth from a dining table, which are tiny movements and similar to each others. However, other daily activities such as walking or standing involved large body movements (e.g., swing arms, bending over) might conduct more intensive vibrations in the frequency domain of CSI measurements. Motivated by this, we then examine to use the Fuzzy C-Means Clustering (FCM) approach to assort the data into two clusters based on two-dimensional features including 


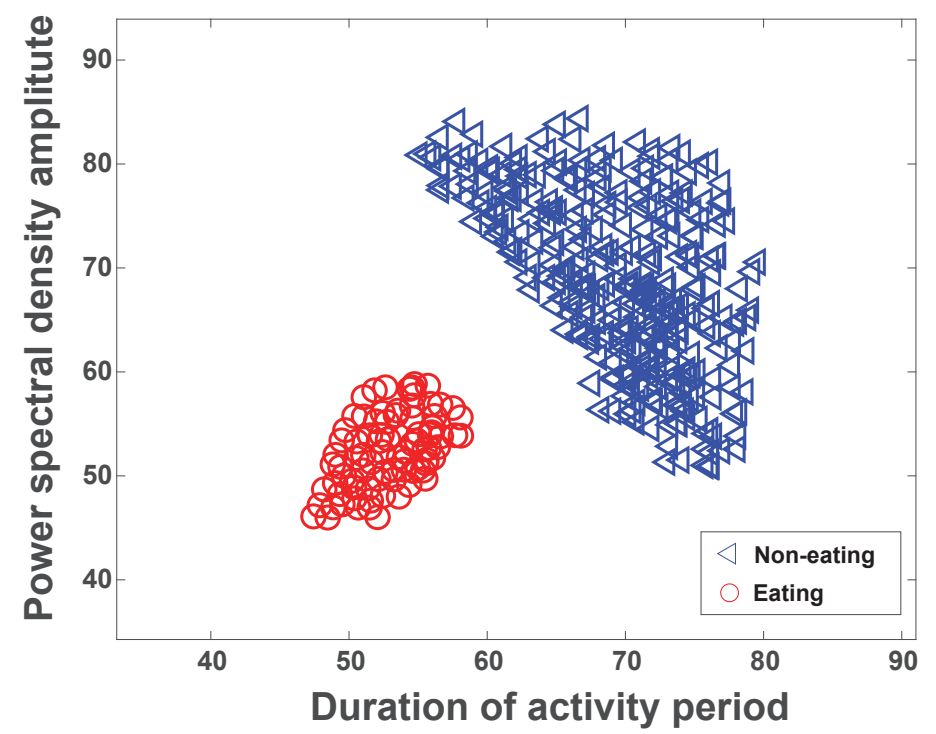

Fig. 4. Fuzzy C-Means cluster results of eating activity and non-eating activity.

the PSD Amplitude and corresponding frequency of these activities. The FCMbased Clustering could be described as:

$$
\Upsilon_{m}=\sum_{i=1}^{A} \sum_{j=1}^{N} \mu_{i j}^{m}\left\|A_{i}-C_{j}\right\|^{2},
$$

where $A$ denotes the number of human activity segments, $N$ is the number of clusters, $m$ denotes the fuzzy partition matrix exponent of controlling the degree of fuzzy overlap, $A_{i}$ represents the $i$ th activity segments, $C_{j}$ denotes the center of the $j$ th cluster, $\mu_{i j}$ represents the degree of membership of $A_{i}$ in the $j$ th cluster.

In order to understand the distribution of participants dietary activities and other daily activities, we first ran a formative study with 2 participants to perform 10 different motions in lab environment. Participants were requested to eat a variety of foods including chips, pizza, bread and noodles with their bare hands, forks, chopsticks. Moreover, we ask participant to perform some non-eating activity including walking, standing, sitting, talking, reading, typing, stretching, respectively. Figure 4 shows the clustering results regarding eating activities and non-eating activities. We note that the proposed approach could successful differentiate the activities into two clusters. In addition, we observe the eating activities are mainly gathered in the lower range of duration period than non-dietary activities are located in the higher range. This is because during the ingestion period, participants keep bringing food to mouth in a repetitive manner, and the eating motions performed by participants usually take faster than other non-dietary activities such as the walking. Based on the differenti- 


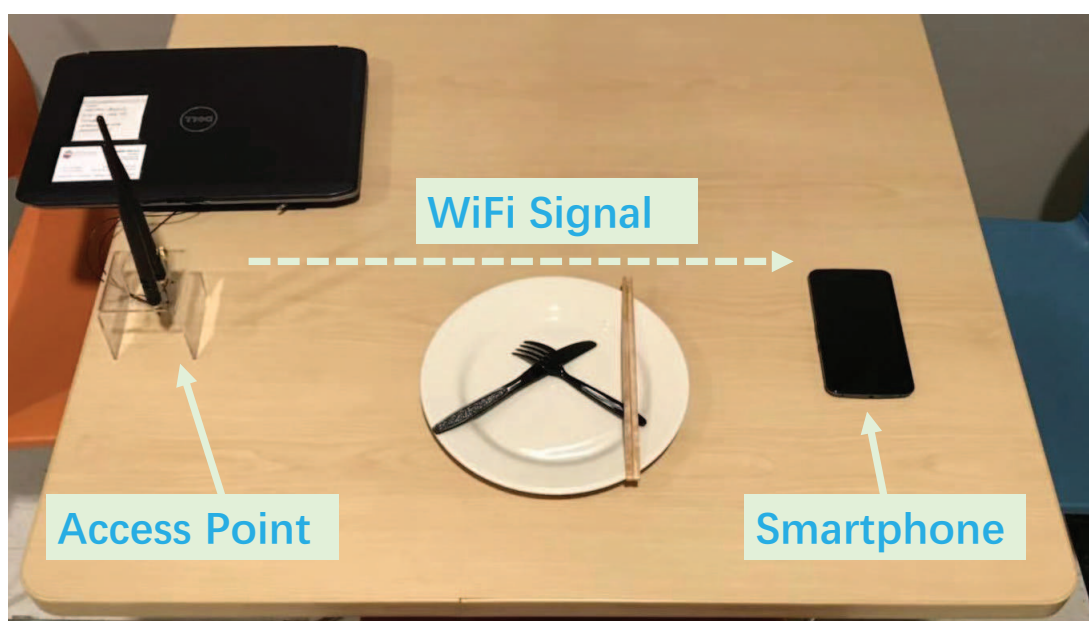

Fig. 5. Illustration of our experimental setting.

ate results, we thus can utilize the derived dietary activities to further process fine-grained classification in terms of the utensils held by users.

\subsection{Food Intake Gesture Classification}

In our system, recognizing various food intake gesture is essential for deriving find-grained statistic information and further detecting eating moments. Among this section, we adopt various machine learning classifiers to identify different eating gestures. We first extract a series of features both from time and frequency domains from thirty subcarriers and then derive two-dimensional vectors as the inputs of multiple classifiers. In order to motivate the suitability of the proposed method with different machine learning algorithm, we apply four kinds of typical machine learning methods (i.e., Random Forest (RF), Naive Bayes (NB), K Nearest Neighbors (KNN), and Discriminant Analysis Classifier (DAC)) to recognize various eating gestures according to the utensils held by the user, including spoon, fork, fork\&knife, hand and chopsticks. The machine learning algorithms are implemented based on the Statistics and Machine Learning Toolbox of Matlab R2019a. We further evaluate the performance of the four traditional classifiers and discuss the results in Section 4.2.

\subsection{Eating Moment Monitoring}

Our intuition of monitoring the eating moment for users is to further estimate the accurate ingestion duration between the interval of each separate food intake gestures. Study [18] shows that the eating speed is positively associated with body weight-gain, indicating that the fast eating speed might increase the risk of overweight and obesity. Thus, it is essential to obtain the fine-grained ingestion period statistics (e.g., the duration time for participants to eat with fork, spoon 


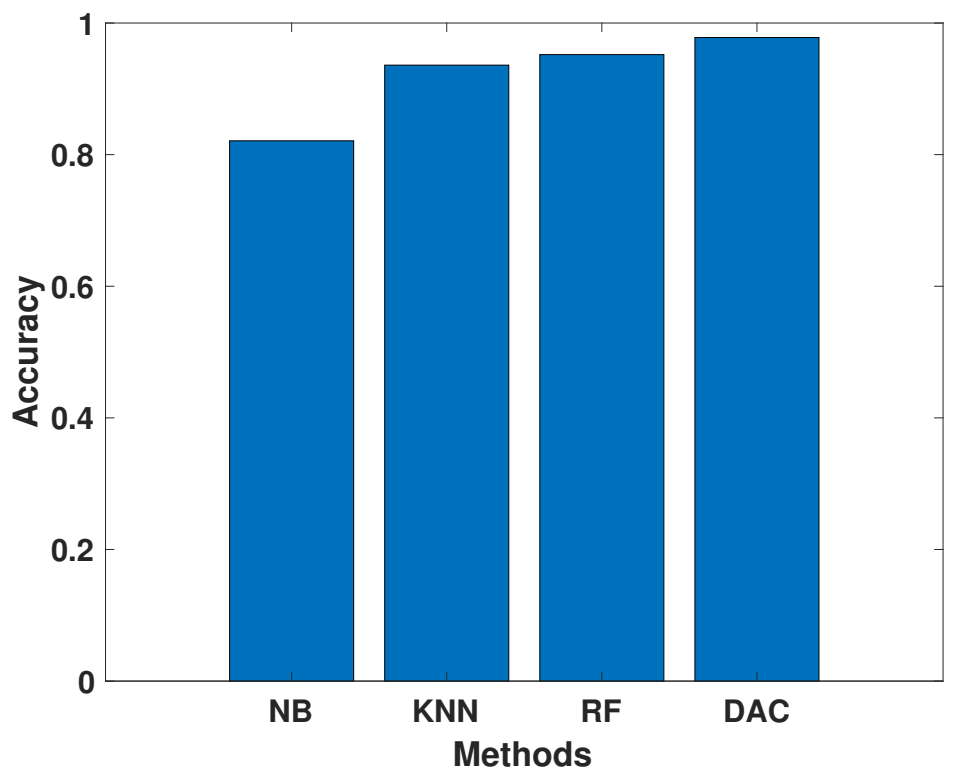

Fig. 6. Comparison of performance among four kinds of machine learning classifiers for food intake gestures identification.

or chopsticks) to further elaborate and evaluate the user's eating behaviors. Given that intuition, we adopt the intake gesture density derivation method to infer the eating moment based on calculating the density of detection food intake gestures when applying a sliding window in a specific eating period length. To compare the prediction estimated dietary duration with the ground truth, we evaluate the detailed results of eating moment monitoring in Section 4.3.

\section{Performance Evaluation}

\subsection{Experimental Methodology}

As shown in Figure 5, we implement our system with a pair of WiFi-enabled devices, including a smartphone and a laptop. Our system aims to imitate the real scenario when people are eating and placing their smartphones on the dining table. In the system, user's smartphone will send WiFi signals to the access point and sense different eating activities. To conduct experiments, we use a Nexus 6 smartphone powered by a $2.7 \mathrm{GHz}$ quad-core Snapdragon 805 processor with 3 GB of RAM as the transmitter. For the receiver, we use a Dell E6430 equipped with Intel $5300802.11 \mathrm{n} \mathrm{WiFi} \mathrm{wireless} \mathrm{card} \mathrm{and} \mathrm{6dBi} \mathrm{rubber} \mathrm{ducky} \mathrm{external}$ omni-directional antennas for extracting CSI readings [19]. The laptop which serves as the access point is configured to run in the netlink mode. Internet Control Message Protocol (ICMP) echo is sent from the laptop and replied by the smartphone to collect the CSI data [20]. In total, 10 volunteers are recruited 


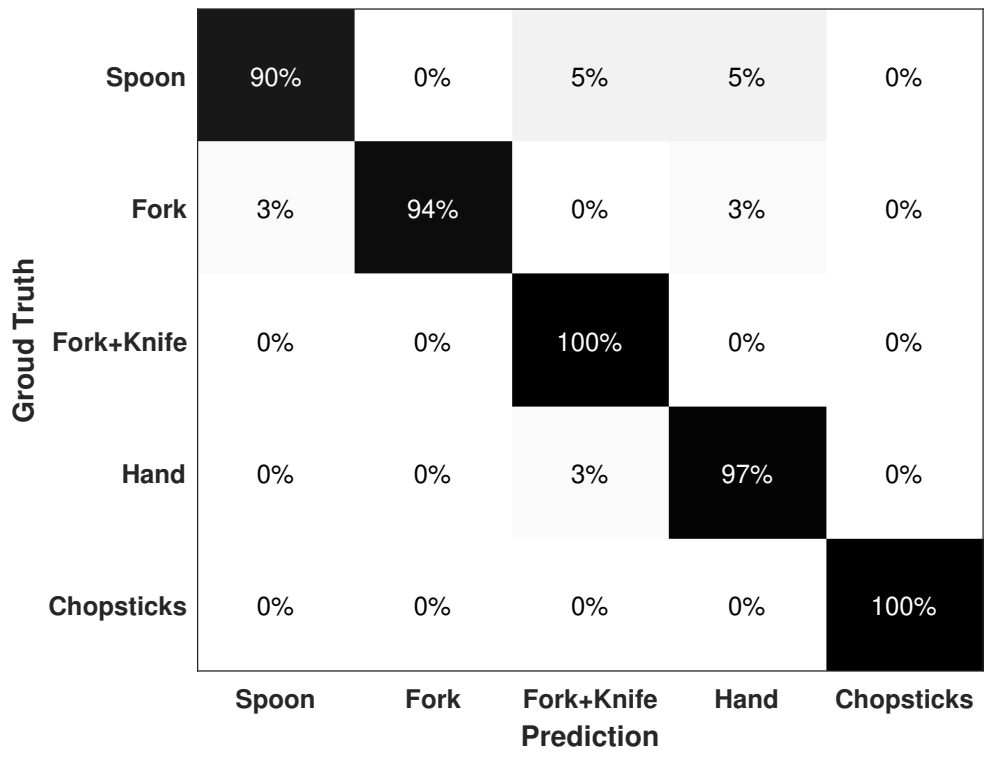

Fig. 7. Confusion matrix of food intake gestures classification.

as the subjects to take part in the experiments and finally we collect 400 minute length data for eating episode. Moreover, the distance between the laptop and the smartphone is $80 \mathrm{~cm}$. Unless mentioned otherwise, half of the collection data is used for training and the rest for testing.

\subsection{Performance of Food Intake Gesture Classification}

We first compare the performance of food intake gesture classification under four typical machine learning classifiers including Random Forest (RF), Naive Bayes (NB), K Nearest Neighbors (KNN), and Discriminant Analysis Classifier (DAC). The parameters of each classifier are tuned to achieve the best performance. As shown in Figure 6, for eating gesture recognition, all the classifiers achieve average accuracy over $80 \%$, indicating that our model could perform well with various classifiers. Specifically, NB, KNN, RF and DAC have average accuracy of $82.1 \%, 93.6 \%, 95.2 \%, 97.8 \%$, respectively. We observe that DAC achieves the best eating gesture identification results. For each eating gesture as depicted in Figure 7, the DAC achieves average accuracy of $90 \%, 94 \%, 100 \%, 97 \%$ and $100 \%$ for five eating gestures (i.e., spoon, fork, fork\&knife, hand, chopsticks). The experimental results show that various eating gestures can all be well recognized by the proposed system. Furthermore, we evaluate the impact of different training sizes on the eating gesture recognition. Figure 8 shows that our system could achieve over $80 \%$ accuracy under different training sizes and even 7 eating motions is sufficient to produce over $80 \%$ accuracy, which further confirms the effectiveness of our system. 


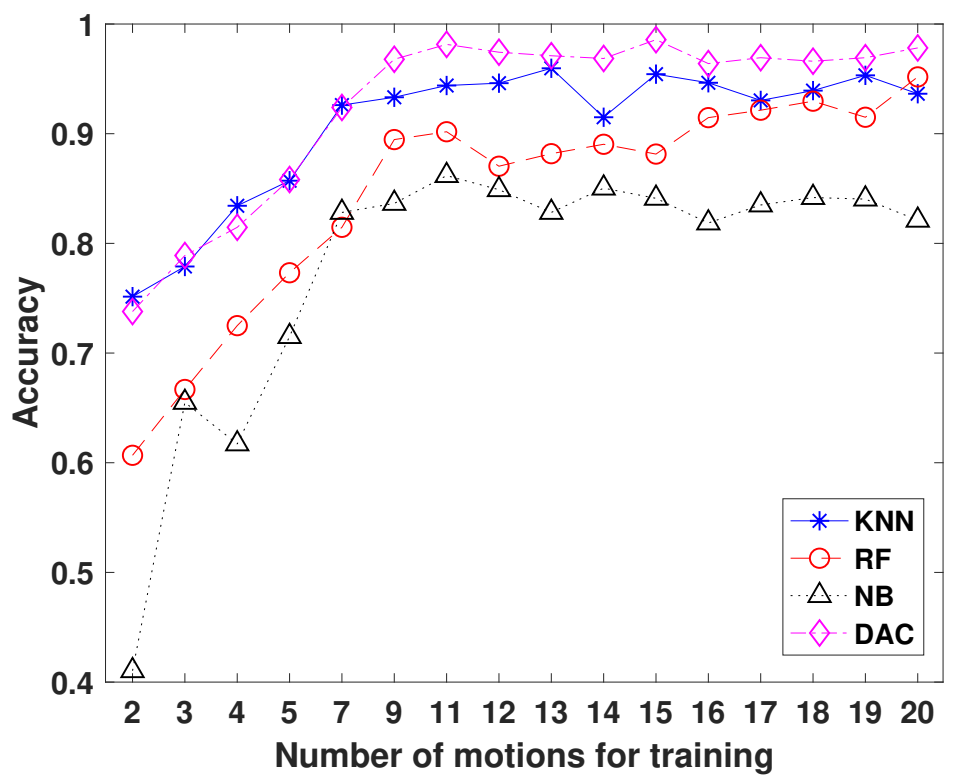

Fig. 8. The impact of training size on the eating gesture recognition.

\subsection{Performance of Eating Moment Monitoring}

Coarse Eating Moment Recognition To determine the starting point and the ending point of eating moment, we first evaluate our system performance on distinguishing eating activities from non-eating activities. Figure 9 provides a detailed picture of the FCM-based cluster performance with different $m$ value, where $m$ denotes the fuzzy partition matrix exponent of controlling the degree of fuzzy overlap. As shown in the figure, our system achieves an accuracy over $90 \%$ when $m$ is over 1.6. We also note that our system achieves $95 \%$ accuracy on eating and non-eating activity recognition given an $m$ value of 2.4. This is because $m$ represents the average coefficient related to the distribution clustering results and enlarging value of $m$ could convey more controlling degree to maximize the distances between different clusters and minimize the inner distance of clusters regarding the eating activities and non-eating activities.

Intake Gesture Moment Estimation Then we evaluate the performance of dietary moment recognition for different food intake gestures. In our experiments, each participant is requested to eat a variety of foods with five kinds of gestures for multiple times. In particular, each gesture will be repeated for 40 times and we collected 200 eating activities for each person in total. As shown in Table 1, the average estimated time duration of using spoon, fork, fork\&knife, hand, and chopsticks are $5 \mathrm{~m} 43 \mathrm{~s}, 5 \mathrm{~m} 34 \mathrm{~s}, 7 \mathrm{~m} 57 \mathrm{~s}, 6 \mathrm{~m} 37 \mathrm{~s}, 6 \mathrm{~m} 34 \mathrm{~s}$ respectively. While the corresponding ground truths are $5 \mathrm{~m} \mathrm{20s,} 5 \mathrm{~m} 52 \mathrm{~s}, 8 \mathrm{~m} \mathrm{11s}, 5 \mathrm{~m} 53 \mathrm{~s}, 6 \mathrm{~m}$ $18 \mathrm{~s}$ respectively. And the ground truths are measured and verified by camera 


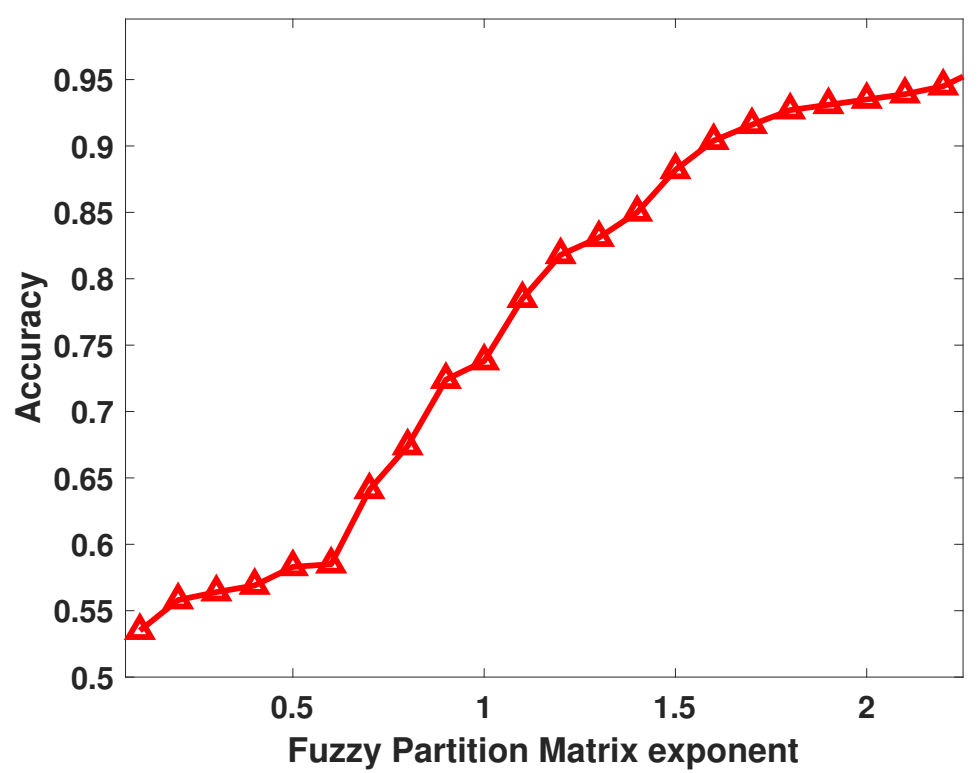

Fig. 9. The performance of dietary activity extraction under various fuzzy partition matrix exponent of FCM clustering.

based method during the experiment. We find that the estimated error of five intake gestures are $23 s, 18 s, 14 s, 44 s$, and $16 s$. In particular, the average duration error of one single eating gesture is within 1.1s. Through analyzing the time duration of each eating gesture, we can have a comprehensive understanding of the dietary moment for each user. Based on the derived fine-grained dietary statistics, it is easy to infer other high-level information such as the estimation of calories intake, analysis of nutritional balance. In addition, the detailed dietary information can also be used to assist various health related problems such as cardiovascular diseases, diabetes, stomach cancers.

Table 1. The average duration of each eating gesture across all users.

\begin{tabular}{|c|c|c|c|c|}
\hline $\begin{array}{c}\text { Eating } \\
\text { Gesture }\end{array}$ & $\begin{array}{c}\text { Estimated Eating } \\
\text { Moment }\end{array}$ & $\begin{array}{c}\text { Ground } \\
\text { Truth }\end{array}$ & $\begin{array}{c}\text { Estimated } \\
\text { Error }\end{array}$ & $\begin{array}{c}\text { Average Estimation } \\
\text { Error for Each Gesture }\end{array}$ \\
\hline Spoon & $5 \mathrm{~m} 43 \mathrm{~s}$ & $5 \mathrm{~m} 20 \mathrm{~s}$ & $23 \mathrm{~s}$ & $0.58 \mathrm{~s}$ \\
Fork & $5 \mathrm{~m} \mathrm{34s}$ & $5 \mathrm{~m} 52 \mathrm{~s}$ & $18 \mathrm{~s}$ & $0.45 \mathrm{~s}$ \\
Fork\&Knife & $7 \mathrm{~m} 57 \mathrm{~s}$ & $8 \mathrm{~m} 11 \mathrm{~s}$ & $14 \mathrm{~s}$ & $0.35 \mathrm{~s}$ \\
Hand & $6 \mathrm{~m} 37 \mathrm{~s}$ & $5 \mathrm{~m} 53 \mathrm{~s}$ & $44 \mathrm{~s}$ & $1.1 \mathrm{~s}$ \\
Chopsticks & $6 \mathrm{~m} \mathrm{34s}$ & $6 \mathrm{~m} 18 \mathrm{~s}$ & $16 \mathrm{~s}$ & $0.4 \mathrm{~s}$ \\
\hline
\end{tabular}




\section{Conclusion}

In this paper, we explore the feasibility of leveraging WiFi signals from smartphones to automatically monitor the fine-grained eating moments. We show that the channel state information extracted from a user's smartphone could be utilized to detect eating activity and further identify different food intake gestures based on the utensils held by users. The statistical dietary information could be used to interpret the detailed intake duration and utensil types during eating period. It also shows the potential to provide the comprehensive understanding with users regarding their eating behaviors and help them to build a healthy dietary pattern. We develop a device-free system, which first differentiates eating activities from non-eating activities based on a fuzzy c-means clustering method. We then utilize the learning-based methods to classify the user's food intake gestures according to the utensils held by the user with four different classifiers (i.e., Random Forest, Naive Bayes, K Nearest Neighbors and Discriminant Analysis Classier). Furthermore, we derive the food intake duration and further estimate the eating moments for each food intake gesture. Extensive experimental results with 10 subjects over 400-minute eating period show that our system can recognize the user's food intake gestures with up to $97.8 \%$ accuracy and estimate the dietary moments within 1.1-second error.

Acknowledgment. This work was supported by the National Science Foundation Grant CNS-1826647.

\section{References}

1. Mokdad, A.H., Ford, E.S., Bowman, B.A., Dietz, W.H., Vinicor, F., Bales, V.S., Marks, J.S.: Prevalence of obesity, diabetes, and obesity-related health risk factors, 2001. Jama 289(1), 76-79 (2003)

2. Organization, W.H.: What are the health consequences of being overweight? https://www.who.int/features/qa/49/en//, accessed April 17, 2019

3. Organization, W.H.: Infobase page for illustrating overweight \& obesity diseases. https://www.who.int/news-room/fact-sheets/detail/obesity-and-overweight/, accessed April 17, 2019

4. Loewy, J.: My macros+ — diet and calories. https://itunes.apple.com/us/app/mymacros-diet-calories/id475249619/, accessed April 21, 2019

5. Feel great about what you ate. https://youate.com/ (2019)

6. Hill, R., Davies, P.: The validity of self-reported energy intake as determined using the doubly labelled water technique. British Journal of Nutrition 85(4), 415-430 (2001)

7. Sazonov, E., Schuckers, S., Lopez-Meyer, P., Makeyev, O., Sazonova, N., Melanson, E.L., Neuman, M.: Non-invasive monitoring of chewing and swallowing for objective quantification of ingestive behavior. Physiological measurement 29(5), 525 (2008)

8. Thomaz, E., Essa, I., Abowd, G.D.: A practical approach for recognizing eating moments with wrist-mounted inertial sensing. In: Proceedings of the 2015 ACM International Joint Conference on Pervasive and Ubiquitous Computing. pp. 10291040. ACM (2015) 
9. Guo, X., Liu, J., Shi, C., Liu, H., Chen, Y., Chuah, M.C.: Device-free personalized fitness assistant using wifi. Proceedings of the ACM on Interactive, Mobile, Wearable and Ubiquitous Technologies 2(4), 165 (2018)

10. Liu, J., Wang, Y., Chen, Y., Yang, J., Chen, X., Cheng, J.: Tracking vital signs during sleep leveraging off-the-shelf wifi. In: Proceedings of the 16th ACM International Symposium on Mobile Ad Hoc Networking and Computing (ACM MobiHoc). pp. 267-276 (2015)

11. Wang, Y., Liu, J., Chen, Y., Gruteser, M., Yang, J., Liu, H.: E-eyes: device-free location-oriented activity identification using fine-grained wifi signatures. In: Proceedings of the 20th annual international conference on Mobile computing and networking. pp. 617-628. ACM (2014)

12. Willett, W.: Nutritional epidemiology, vol. 40. Oxford University Press (2012)

13. Sun, M., Fernstrom, J.D., Jia, W., Hackworth, S.A., Yao, N., Li, Y., Li, C., Fernstrom, M.H., Sclabassi, R.J.: A wearable electronic system for objective dietary assessment. Journal of the American Dietetic Association 110(1), 45-47 (2010)

14. O'Loughlin, G., Cullen, S.J., McGoldrick, A., O'Connor, S., Blain, R., O'Malley, S., Warrington, G.D.: Using a wearable camera to increase the accuracy of dietary analysis. American journal of preventive medicine 44(3), 297-301 (2013)

15. Hodges, S., Williams, L., Berry, E., Izadi, S., Srinivasan, J., Butler, A., Smyth, G., Kapur, N., Wood, K.: Sensecam: A retrospective memory aid. In: International Conference on Ubiquitous Computing. pp. 177-193. Springer (2006)

16. Bedri, A., Li, R., Haynes, M., Kosaraju, R.P., Grover, I., Prioleau, T., Beh, M.Y., Goel, M., Starner, T., Abowd, G.: Earbit: using wearable sensors to detect eating episodes in unconstrained environments. Proceedings of the ACM on interactive, mobile, wearable and ubiquitous technologies 1(3), 37 (2017)

17. Zhang, S., Ang, M., Xiao, W., Tham, C.K.: Detection of activities by wireless sensors for daily life surveillance: eating and drinking. Sensors 9(3), 1499-1517 (2009)

18. Ohkuma, T., Hirakawa, Y., Nakamura, U., Kiyohara, Y., Kitazono, T., Ninomiya, T.: Association between eating rate and obesity: a systematic review and metaanalysis. International journal of obesity 39(11), 1589 (2015)

19. Halperin, D., Hu, W., Sheth, A., Wetherall, D.: Tool release: gathering $802.11 \mathrm{n}$ traces with channel state information. ACM SIGCOMM Computer Communication Review 41(1), 53-53 (2011)

20. Li, M., Meng, Y., Liu, J., Zhu, H., Liang, X., Liu, Y., Ruan, N.: When csi meets public wifi: Inferring your mobile phone password via wifi signals. In: Proceedings of the 2016 ACM SIGSAC Conference on Computer and Communications Security. pp. 1068-1079. ACM (2016) 